\title{
Discours
}

Revue de linguistique, psycholinguistique et

informatique. A journal of linguistics, psycholinguistics and computational linguistics

$23 \mid 2018$

Varia

Cohésion, cohérence et digression dans le discours à dominante explicative : une perspective diachronique (de la fin du XIII ${ }^{\mathrm{e}}$ au XVI ${ }^{\mathrm{e}}$ siècle)

\section{Sabine Lehmann}

\section{OpenEdition}

\section{Journals}

Édition électronique

URL : http://journals.openedition.org/discours/9922

DOI : $10.4000 /$ discours.9922

ISSN : 1963-1723

Éditeur :

Laboratoire LATTICE, Presses universitaires de Caen

\section{Référence électronique}

Sabine Lehmann, "Cohésion, cohérence et digression dans le discours à dominante explicative : une perspective diachronique (de la fin du XIII au XVIe siècle) », Discours [En ligne], 23 | 2018, mis en ligne le 21 décembre 2018, consulté le 13 juin 2019. URL : http://journals.openedition.org/discours/9922 ; DOI : 10.4000/discours.9922 

Revue de linguistique, psycholinguistique et informatique

\section{Cohésion, cohérence et digression}

dans le discours à dominante explicative :

une perspective diachronique

(de la fin du XIII' au XVI ${ }^{e}$ siècle)

Sabine Lehmann

Laboratoire MoDyCo

Université Paris Ouest Nanterre La Défense

Sabine Lehmann, «Cohésion, cohérence et digression dans le discours à dominante explicative: une perspective diachronique (de la fin du XIII au XVle siècle)», Discours [En ligne], 23 | 2018, mis en ligne le 21 décembre 2018.

URL: http://journals.openedition.org/discours/9922

Titre du numéro: Varia

Coordination: Saveria Colonna \& Sarah Schimke

Date de réception de l'article: 20/07/2018

Date d'acceptation de l'article: 03/12/2018 



\title{
Cohésion, cohérence et digression dans le discours à dominante explicative : une perspective diachronique (de la fin du XIII' au XVI' siècle)
}

\author{
Sabine Lehmann \\ Laboratoire MoDyCo \\ Université Paris Ouest Nanterre La Défense
}

\begin{abstract}
La digression fait partie d'une série de mouvements textuels qui interrompent le discours en produisant une rupture de la chronologie du texte, une insertion que l'on compare parfois à une parenthèse. Dans cette série, la digression coexiste avec l'exemplum et l'illustration. C'est l'effet de déviation mis en place dans le tissu textuel qui justifie ce regroupement. Dans cet article, nous montrons que la digression relève toujours d'une stratégie qui contribue à l'instauration d'effets de cohésion et de cohérence; elle s'inscrit dans le mouvement de complexification des structures textuelles et séquentielles observable dans le parcours diachronique du français. Le cadre diachronique mis en place dans notre étude est construit autour de la période du moyen français, caractérisée par l'apparition de textes à dominante explicative ou argumentative. Le développement de ces nouveaux modèles textuels va de pair avec une nouvelle conception de la cohérence discursive. C'est dans ces nouveaux types de textes représentatifs de domaines de savoir variés (par exemple médecine, philosophie, agriculture) que nous étudions les modalités d'inscription de la digression. Ces dernières peuvent être de nature implicite et explicite (marqueurs permettant de cerner le début et / ou la fin d'une digression). Les marqueurs convoqués permettent de ponctuer le discours et de contribuer ainsi à sa stratification.
\end{abstract}

Mots clés: cohésion, cohérence, digression, exemplum, séquence

Digression is a type of textual movement that interrupts the flow by disrupting the chronology of the text. The effect of the insertion may be compared to that of parentheses within the textual flow. Digression may be considered similar to the exemplum and the illustration. The similarity lies in the effect of the diversion introduced into the fabric of the text. In this article, we show that digression always indicates a strategy that contributes to the creation of cohesion and coherence effects. It is part of the move towards complexity of the textual and sequential structures observable in the development of French over time. The timeframe established in our study is built around the Middle French period, which is characterized by the appearance of texts that are predominantly explanatory or in support of an argument. The development of these new types of text goes hand in hand with a new concept of discursive coherence. It is in these new types of text representing diverse areas of knowledge (for example medicine, philosophy, agriculture) that we are studying the methods by which the digression is inserted. These methods might be of an implicit or explicit nature (with markers allowing the reader to detect the start and/or end of a digression). The markers used allow the discourse to be punctuated and hence contribute to its stratification.

Keywords: cohesion, coherence, digression, exemplum, sequence 


\section{Introduction}

Dans le cadre de notre étude nous nous intéresserons à la digression en tant que fonctionnement discursif analysé du point de vue de sa contribution à la mise en place d'effets de cohésion et de cohérence ainsi que de celui de la complexification des structures textuelles et séquentielles. Le cadre diachronique établi dans cette étude est construit autour de la période du moyen français - période pendant laquelle se développe le texte explicatif/argumentatif en langue française. Ces nouveaux types de textes se caractérisent par une autre conception de la cohérence discursive et, par voie de conséquence, une autre organisation du niveau thématique. Les exemples étudiés sont extraits d'ouvrages à dominante explicative/ informative/ argumentative ${ }^{1}$ et représentatifs des différents domaines de savoir (par exemple: traités d'éducation, de médecine, de philosophie, d'agriculture) auxquels le nouveau lectorat se constituant à partir de la fin du XIII siècle aura accès grâce aux ouvrages de vulgarisation scientifique qui lui sont destinés.

Après avoir analysé dans un premier temps la relation entre les deux notions inséparables de cohésion et cohérence, d'une part, et celle de digression d'autre part, nous procéderons dans un second temps à l’analyse de la stratégie digressive dans différents textes à dominante explicative et/ou argumentative. Notre approche, consistant à étudier la digression comme une mise en scène d'une frontière textuelle, comme un dispositif dont on tente de repérer les divers signaux, les arguments clés, les constantes thématiques, les stratagèmes et les enjeux, renforce l'idée de la nécessité d'analyser la digression en termes de stratégie discursive (Sabry, 1992). Il s'agit pour nous de démontrer que cette stratégie peut être au service de la cohésion/cohérence. Son usage peut permettre de renforcer la cohésion aussi bien d'une matière sujette aux égarements du plaisir de raconter que d'un discours «sérieux», écrit par un auteur au service de la transmission d'un savoir et se livrant de plus en plus à des considérations qui se détachent du récit pour s'épanouir de façon autonome. Cette autonomisation est comparable à celle des passages de second plan étudiée par Combettes (2012a) et produisant dès le XIV siècle des passages descriptifs caractérisés par une organisation interne qui les rend en quelque sorte indépendants du contexte narratif dans lequel ils s'insèrent.

1. Dans Le texte informatif: aspects linguistiques, Combettes et Tomassone reconnaissent que tout texte est, à un certain degré, informatif et que «le terme d'expositif serait sans doute meilleur que celui d'informatif, relativement vague» (Combettes et Tomassone, 1988: 6). Tandis que le type argumentatif vise à modifier des croyances, des représentations, le texte informatif-expositif vise moins à transformer des convictions qu'à apporter un savoir. Combettes et Tomassone opèrent également une distinction entre informer-exposer, d'une part, et expliquer, d'autre part: «Expliquer nous semble constituer une intention particulière qui ne se confond pas avec informer; le texte explicatif a sans doute une base informative, mais se caractérise, en plus, par la volonté de faire comprendre les phénomènes [...]. Le texte informatif, en revanche, ne vise pas à établir une conclusion: il transmet des données, certes organisées, hiérarchisées [...], mais pas à des fins démonstratives. Il ne s'agit pas, en principe, d'influencer l'auditoire, de le conduire à telle ou telle conclusion, de justifier un problème qui serait posé» (Combettes et Tomassone, 1988: 6). 
La digression s'éloigne d'une «matière» en termes d'espace comme si le texte produit était une des formes possibles d'un texte idéal, perdu à chaque détour, et dont on ne fait que s'approcher en reprenant le fil narratif ou explicatif. Les errances discursives que représentent les digressions produisent un discours amplifié, obligeant le lecteur à quitter la trame principale (narrative, explicative ou argumentative) mais tout en lui permettant de découvrir des richesses inattendues. L'inscription de la digression dans l'espace séquentiel provoque des ruptures plus ou moins marquées par des signaux posés au seuil de la digression et à sa fin. La pratique digressive produit une rupture temporaire dans la logique principale et «relève de ce qui est en marge de la rigueur, mais garde un lien certain avec le sujet» (Ueltschi, $2005: 400$ ). Ce lien certain avec le sujet s'impose afin de garantir la cohérence de l'ensemble. L'idée de rupture du discours caractérise également l'exemplum et l'illustration. C'est l'effet commun d'une déviation mise en place dans le tissu textuel qui justifie le regroupement des trois termes. Mais, contrairement à la digression, l'exemplum et l'illustration se situent dans l'axe logique de l'argumentation dont ils doivent expliciter la portée concrète par des parenthèses brèves et narratives. Dans le cadre de notre étude, nous nous intéresserons à la parenté entre l'exemplum et la digression dans la mesure où le discours scientifique de la fin du Moyen Âge convoque les deux procédés pour offrir des divertissements, des enseignements, des leçons. Dans les deux cas, les séquences traversées par ces réseaux de déviation sont caractérisées par une complexification des structures discursives et thématiques. Nous placerons donc l'analyse de la tension entre le discours explicatif/informatif linéaire et les digressions au niveau de la séquence - espace discursif hétérogène par excellence qui contribue, à travers sa dépendance par rapport à l'ensemble textuel, à la mise en place d'effets de cohésion et de cohérence. L'emploi des qualificatifs «explicatif» et «argumentatif» renvoie donc aux différents modèles séquentiels élaborés par JeanMichel Adam (1992) en réaction à la trop grande généralité des typologies de texte apparues avec les grammaires de texte. La théorie des séquences considère qu'un petit nombre de types de séquences de base guide les empaquetages prototypés de propositions qui forment les diverses macro-propositions (narratives, descriptives, explicatives, argumentatives, dialogales).

Afin de pouvoir identifier les digressions au sein des espaces séquentiels, nous sommes partie de notre expérience de lectrice d'ouvrages appartenant à la littérature didactique de la fin du Moyen Âge et selon laquelle les digressions peuvent être implicites ou marquées explicitement par des paradigmes introducteurs (par exemple: par especial) ou des béquilles argumentatives (item, nota). Les exemples cités pour les matérialisations explicites résultent de l'interrogation de la base textuelle du Dictionnaire du moyen français $(D M F)$ à partir de la liste des signaux explicites mentionnés par Ueltschi (2005: 396-397). Nous avons également ajouté des textes qui ne font pas partie de la base du $D M F$ mais à partir desquels nous menons nos études centrées sur l'évolution de la textualité au Moyen Âge (plus particulièrement des textes explicatifs, comme, par exemple, La cbirurgie d'Henri de Mondeville ou Le théâtre d'agriculture d'Olivier de Serres). L'identification des 
digressions implicites a été réalisée surtout dans les textes lus et étudiés en intégralité (notamment Le mesnagier de Paris et les textes de médecine et d'agriculture de la fin du Moyen Âge) ainsi qu'à partir d'une interrogation du contexte élargi dans lequel apparaissent les marqueurs explicites. L'objectif de notre étude est donc de donner un premier aperçu de la relation qu'entretient la digression avec la cohésion et la cohérence des textes explicatifs en moyen français. Cette approche qui s'inscrit dans le domaine de la linguistique textuelle diachronique ne représente qu'un point de départ pour des investigations ultérieures qui concernent aussi bien l'aspect quantitatif (exploitation plus systématique des textes pour fournir des données quantifiées) que qualitatif (élargissement de la liste de moyens mis en œuvre).

\section{Cohésion, cohérence et digression}

La cohérence est, en linguistique textuelle, inséparable de la notion de cohésion avec laquelle elle est souvent confondue. Afin d'aboutir à ce qu'Adam et Heidmann (2004: 62) appellent «un effet de texte», un texte doit répondre aux exigences imposées par ces deux fonctionnements textuels fondamentaux. Le terme «cohésion» désigne, depuis Cohesion in English de Halliday et Hasan $(1976)^{2}$, l'ensemble des moyens linguistiques qui assurent les liens intra- et interphrastiques permettant à un énoncé d'apparaître comme un texte. Relevant de la texture, la cohésion est un aspect de la grammaticalité, un dispositif assuré par «l'emploi de procédés linguistiques appropriés ([...] choix de l'article défini/indéfini, pronominalisation, anaphores, connecteurs, etc.)» (Ducrot et Schaeffer, $1995: 503)$. Adam (1992: 22) précise qu'«à un niveau intermédiaire entre le global et le local, la dimension sémantico-référentielle est analysable en termes d'isotopie(s) et de cohésion du monde représenté». Effectivement, les marqueurs de connexité agissant à ce niveau induisent un effet sémantique de cohésion qui permet de dégager le thème général du texte.

Rappelons que les connexions qui s'établissent au sein d'un texte sont à la fois structurales et non structurales. À côté des liens structuraux (dispositif syntaxique), il existe, dans les différentes langues et aux différentes phases de leur évolution, un ensemble d'outils relationnels de nature sémantico-pragmatique qui, en quelque sorte, complètent le système des relations distributionnelles ainsi que le dispositif logico-énonciatif (thème/propos). Parmi ces marques on trouve notamment les connecteurs, les différentes formes d'anaphores qui assurent les solidarités référentielles et donnent naissance à des chaînes de référence, les expressions introductrices de cadres de discours (domaines ou cadres temporels, spatiaux, modaux, etc.) ainsi

2. «Le concept de cohésion est apparu sous son acception actuelle vers la fin des années soixante dans les travaux des linguistes anglophones (Halliday, Huddleston, Quirk et l'école firthienne de Londres, Bolinger et les néo-structuralistes américains). C'est probablement parce que l'anglais a un système de marques flexionnelles très réduit (et qui ne cesse de se réduire) que s'y pose de façon insistante la question de la cohésion, qui peut se définir comme l'ensemble des relations signalant le texte (restreint parfois à une phrase) en tant qu'entité sinon linguistique, du moins sémantique, communicative, fonctionnelle, empirique ou pragmatique» (Juillard, 2005: 175). 
que les marques configurationnelles (alinéas, organisateurs métadiscursifs) qui délimitent au sein du continuum textuel des ensembles présentés par le locuteur comme constituant une unité. C'est sur cet ensemble de marques que reposent les liens de cohésion qui contribuent à ce que Halliday et Hassan (1976) appellent la «texture» d'un ensemble textuel. Ce système de marques constitue un dispositif regroupant les différents niveaux d'organisation du discours (de la syntaxe au niveau configurationnel en passant par les outils relationnels et la progression thématique) qui «peuvent être envisagés séparément, mais surtout [dont la] distinction permet d'étudier leurs interactions» (Charolles, 1995: 128).

Dans le but d'«articuler l'unité contradictoire du texte, objet formel abstrait, et du discours, pratique sociale concrète», Slakta (1975: 30) considère que la cohésion se détermine linguistiquement au plan de l'ordre (interne et abstrait) du texte. Cet ordre se manifeste aux niveaux global et local. Selon Beaugrande (1979: 490), la cohésion correspond à une «textualité basée sur la forme» sous laquelle se présente l'ensemble textuel aux niveaux micro- et macrostructurel; la cohérence, en revanche, est définie comme une «textualité basée sur l'information».

Comme nous l'avons déjà signalé, au départ la cohérence, propriété définitoire du texte (elle est ce qui fait qu'un texte est perçu comme un texte, c'est-à-dire un ensemble énoncé qui se tient), a été souvent confondue avec sa matérialisation, l'ensemble du dispositif linguistique responsable de l'effet de cohésion. Mais la cohérence n'est pas une propriété «strictement linguistique des textes», elle résulte d'un jugement qui prend appui sur la connaissance de la situation et les savoirs lexico-encyclopédiques des sujets ${ }^{3}$. D'autres théories insistent sur les conditions logico-sémantiques (Beaugrande, 1979) ou cognitives (Charolles, 1988 et $1995^{4}$ ) de son émergence. En somme, le lien entre moyens et résultat n'est pas biunivoque. Adam (2002: 99) rappelle à juste titre que ce n'est pas seulement parce qu'il présente des marques de connexité-cohésion qu'un texte est jugé cohérent. Dans son article intitulé «Introduction aux problèmes de la cohérence des textes», Charolles décrit la complexité du lien entre cohésion et cohérence:

Dans une grammaire de texte, la base du texte (sa représentation structurelle profonde) est de nature logico-sémantique: les constituants phrastiques, séquentiels et textuel [sic] figurent sous la forme d'une chaîne de représentations sémantiques aménagée de telle sorte que soient manifestées leurs relations de connexité. Les règles de cohérence agissent sur la constitution de cette chaîne, les contraintes qu'elles stipulent portent donc sur des traits (logico) sémantiques c'est-à-dire au bout du compte linguistiques. On montrera cependant que nombre de ces règles (et donc aussi que certains aspects des méta-règles qui en sont leur contrepartie

3. «La cohérence étant, en revanche, de l'ordre des pratiques discursives, elle relève plutôt d'une analyse de discours attentive au genre de discours, à la visée du texte, aux savoirs réciproques des co-énonciateurs dans le contexte d'une interaction donnée» (Charaudeau et Maingueneau, 2002: 100).

4. Charolles $(1988: 55 ; 1995)$ fait du «besoin de cohérence» un principe général d'interprétation, une forme a priori de l'identification d'un ensemble verbal comme texte. 
générale) exigent que soient pris en compte des paramètres pragmatiques renvoyant aux participants à l'acte de communication textuelle. Avec ces règles les grammaires de texte dépassent le simple cadre du texte pour aborder le plan du discours (du texte en situation) ce qui ne manque pas d'accrôitre leur puissance.

(Charolles, 1978: 14)

Dans cet article, Charolles énonce et discute quatre méta-règles de cohérence renvoyant à une appréhension générale, approximative et encore pré-théorique de la question:

I. méta-règle de répétition: «Pour qu'un texte soit (microstructurellement ou macrostructurellement) cohérent, il faut qu'il comporte dans son développement linéaire des éléments à récurrence stricte».

(Charolles, 1978: 14)

II. méta-règle de progression: «Pour qu'un texte soit microstructurellement ou macrostructurellement cohérent, il faut que son développement s'accompagne d'un apport sémantique constamment renouvelé».

(Charolles, 1978: 20)

III. méta-règle de non-contradiction: «Pour qu’un texte soit microstructurellement ou macrostructurellement cohérent, il faut que son développement n'introduise aucun élément sémantique contredisant un contenu posé ou présupposé par une occurrence antérieure ou déductible de celle-ci par inférence».

(Charolles, 1978: 22)

IV. méta-règle de relation: «Pour qu'une séquence ou qu'un texte soient cohérents, il faut que les faits qu'ils dénotent dans le monde représenté soient [directement] reliés». (Charolles, 1978: 31)

À travers ces méta-règles Charolles insiste sur le caractère à la fois linguistique et pragmatique de la cohérence. Le caractère linguistique s'explique à partir du lien étroit qui s'établit entre la cohésion (aspect de la grammaticalité) et la cohérence (aspect de l'acceptabilité). En effet, les marqueurs de cohésion ne sont que des indices d'une cohérence à construire par un travail interprétatif. L'aspect pragmatique se manifeste à travers la formulation d'hypothèses pragmatiques sur la visée du texte, inséparable de sa pertinence situationnelle. Ces deux aspects permettent de placer la notion de cohérence au croisement de la linguistique textuelle et de l'analyse du discours.

Comment appréhender la notion de digression face à ces deux principes qui permettent de transformer un ensemble verbal en texte et dont les fonctionnements sont conditionnés par des contraintes de type ordre et grammaticalité, pour ce qui relève de la cohésion, et acceptabilité, pour ce qui est de la cohérence? Comme l'a souligné Sabry dans l'introduction à son ouvrage Stratégies discursives: digression, transition, suspens, "placer la digression dans un simple rapport cohérence/incohérence, c'est déboucher très vite sur une impasse» (Sabry, 1992: 6). Tandis que les uns voient en elle un facteur potentiel de désorganisation, les autres s'appliquent à aplatir le phénomène 
«digression», soit en le neutralisant, soit en lui conférant - par centralisation - le rôle d'élément moteur et unificateur. Selon nous, ces deux extrêmes se croisent et se neutralisent, d'une certaine façon, dans une approche qui consiste à montrer que la digression relève toujours d'une stratégie. En tant que telle, elle peut, par exemple, être au service du plaisir de la parole (digression-jouissance), poursuivre un objectif pédagogique (digression pédagogique) ou promulguer un savoir emprunté aux ouvrages encyclopédiques. Mais dans tous les cas la digression se présente comme utile, qu'elle cherche à amuser, à séduire, à conseiller, à renseigner ou à engager à l'action.

Dérivée de l'étymon latin digressio - proprement, «action de s'éloigner»-, la digression est une notion rhétorique soumise à la codification depuis l'Antiquité 5 . Dès Aristote, la digression est pensée en termes de stratégie d'éloquence. Elle entre dans les figures du delectare; c'est un des meilleurs moyens de plaire. D'une façon plus générale, la digression relève aussi du movere. Elle est propice à la mise en scène des sentiments et des passions.

Les théoriciens médiévaux, qui classent la digression parmi les procédés de l'amplificatio, en ont proposé des définitions variées qui reflètent le caractère mouvant et protéiforme de la digression. À l'instar de leurs ancêtres médiévaux, les théoriciens contemporains éprouvent des difficultés à faire entrer la digression dans l'appareil terminologique moderne et à écrire sa grammaire:

Le terme et le procédé qu'il désigne restent absents de la terminologie actuelle; en témoignent les dictionnaires de Dubois, de Greimas, de Ducrot et Todorov, qui en revanche accordent une place à d'autres termes de rhétorique comme la métaphore ou la métonymie. Le Dictionnaire de poétique et de rbétorique d'Henri Morier accorde, il est vrai, une rubrique à la digression ${ }^{6}$ et l'on peut noter également le souci qu'a eu Chklovski ${ }^{7}$ de délimiter les différents rôles attribuables à celle-ci et qui, selon lui, seraient au nombre de trois: (1) introduire dans le roman une matière nouvelle, (2) ralentir l'action, la freiner, (3) créer un contraste.

(Sabry, 1992:6)

5. Dans son Institution oratoire, Quintilien définit la digression en ces termes: «Tout ce qui se dit en dehors des cinq parties que nous avons définies (exorde, narration, confirmation, réfutation, péroraison) est une digression : indignation, commisération, détestation, injure, excuse, conciliation, réfutation des propos outrageants. Il en est de même pour tout ce qui n'est pas impliqué dans la question, à savoir toute amplification, toute atténuation, tout genre d'appel aux sentiments, tout passage surtout qui apporte au discours de l'agrément et de l'ornement, et traite du luxe, de l'avarice, de la religion, des devoirs [...]» (Livre IV, 3, 15; cité par Gardes-Tamine, 2002: 101-102).

6. «Digression: partie du discours où l'auteur s'éloigne du sujet pour narrer une anecdote, un souvenir, dépeindre un paysage, un objet d'art, etc., et leur donner un développement inattendu. C'est une histoire en marge de l'histoire. La digression peut répondre à plusieurs intentions: 1. Elle distrait le lecteur d'un sujet trop aride; 2 . Elle peut être une sorte de suspension destinée à faire languir le lecteur dans l'attente impatiente d'un bonheur ou d'un malheur prévu. Prenons garde à la digression: elle fait perdre le fil des événements, rompt l'unité d'action, ennuie parfois le lecteur. Mais elle peut être utile à l'orateur, à l'avocat désireux de créer une diversion, une détente, avant de revenir avec plus de fougue ou de sérénité au sujet traité [...]» (Morier, 1981: s. v. «Digression»). 
Parmi les éléments les plus fondamentaux des définitions du digressif il faut citer les termes «interruption» (ou rupture) et «autre». En effet, la digression est une interruption de propos par l'inscription d'un «discours "autre" (Milhe Poutingon, 2012 : 27). Elle a ce statut paradoxal de l'élément «en marge de», qui est comme décalé par rapport au discours qui l'englobe et lui donne sa raison d'être. Le moment d'interruption correspond à la mise en place d'un nouvel espace discursif qui se désolidarise de l'histoire ou du fil conducteur d'une explication/argumentation pour «parler d'autre chose». Cette déviation est un motif d'hétérogénéité : elle induit une collision entre deux espaces discursifs et provoque une rupture de la cohésion textuelle du discours-cadre ou, autrement dit, de la séquence qui fait émerger la déviation. La rupture peut se manifester à travers une discontinuité temporelle ou un décrochage énonciatif. Cependant, cette hétérogénéité créée par l'insertion d'un fragment digressif est mise en scène au bénéfice d'une continuité discursive. Cette dernière s'inscrit dans le discours sous forme de rattrapages temporels ou énonciatifs ${ }^{8}$ qui gomment les disparités entre les espaces discursifs, ainsi que par le recours à des marqueurs qui signalent la mise en place d'une digression tout en lui accordant une place dans la séquence-cadre. La digression est donc dans un rapport hiérarchique avec son contexte et participe aux faits de stratification du discours.

La relation entre la digression et la cohérence passe par les notions de «lien», de «congruence» et la nécessité de maintenir le détour dans les limites du sujet. C’est en nous appuyant sur les quatre méta-règles définies par Charolles que nous proposons de caractériser la digression comme un écart à la méta-règle dite de relation. Cette dernière est de nature fondamentalement pragmatique. Selon Charolles, "pour qu'une séquence soit admise comme cohérente il est nécessaire que les actions, états ou événements qu'elle dénote soient perçus comme congruents dans le type de monde reconnu par celui qui l'évalue» (Charolles, 1978: 31). Cette relation de congruence est «une relation assez lâche» (par opposition à celle de non-contradiction) parce qu'elle repose uniquement sur l'aperception d'un rapport de faits (cause, conséquence, condition, etc.). Le jugement de cohérence s'appuie sur «la reconnaissance d'un lien de pertinence factuelle» (Charolles, 1978: 32) qui dépend des qualités attribuées au monde interprété et représenté ainsi que du but que s'est fixé l'énonciateur. Dans le cas d'une digression, l'établissement d'une relation de congruence entre les faits dénotés à l'intérieur du monde interprété doit donc rester possible; dans le cas contraire, il s'agirait d'un hors-sujet. La définition proposée par le Gradus a le

8. Dans son article consacré à l'anecdote digressive dans les récits de voyage, Magri-Mourgues a analysé ces phénomènes de rattrapage. Le rattrapage temporel, par exemple, se produit de la façon suivante: «Pour les anecdotes qui introduisent un décalage dans le temps, la clôture de la séquence assure le "rattrapage temporel" par le biais des adverbes encore ou depuis, qui établissent la continuité du passé à l'époque de la narration. On glisse d'un plan d'énonciation à l'autre; il se produit une interaction entre deux époques, le moment de l'histoire et le moment de l'énonciation. L'emploi de ces adverbes temporels indifféremment pour les anecdotes historiques ou légendaires contribue à brouiller les catégories. Par exemple: "On montre encore, sur une pointe de rochers placée près du Nil, un marabout qui est le tombeau du Bellal” (p. 172)» (Magri-Mourgues, $2005: 166$ ). 
mérite (malgré son caractère assez ambigu quant à la nature exacte de la digression) de mettre en valeur les tensions inhérentes à l'emploi d'une digression par rapport au risque du hors-sujet: la digression serait donc «un endroit d'un ouvrage où l'on traite des choses qui paraissent hors du sujet principal, mais qui vont pourtant au but essentiel que s'est proposé l'auteur» (Dupriez, 1984: 157). La digression implique alors une adéquation relative au sujet.

\section{Explication, argumentation et digression}

Comme nous l'avons déjà signalé, c'est pendant la période du moyen français qu'apparaissent des textes dominés par l'intention de transmettre un savoir, d'expliquer ses composantes, d'argumenter afin de convaincre le lecteur. Au niveau du discours, cette intention est matérialisée par des séquences explicatives ou argumentatives permettant au lecteur d'avoir accès à de nouveaux types de savoir qui empruntent leur cadre spatio-temporel au monde et à la vie des hommes.

Dans la séquence explicative de base (Grize, 1981; Coltier, 1986; Adam, 1992), un opérateur de type Pourquoi? ou Comment? (implicite ou explicite) questionne une ou plusieurs composantes d'un savoir ou une représentation problématique de ce dernier. Tandis que l'objectif de l'explication consiste à passer du problème à sa solution-explication, l'argumentation, quant à elle, est orientée vers la persuasion. Cet effet perlocutoire est mis au premier plan par la définition de Perelman et Olbrechts-Tyteca, pour qui «l'objet [de la théorie de l'argumentation] est l'étude des techniques discursives permettant de provoquer ou d'accroître l'adhésion des esprits aux thèses qu'on présente à leur assentiment» (Perelman et Olbrechts-Tyteca, 1976: 59). Pour le nouveau lectorat non savant se constituant à partir de la fin du XIII ${ }^{e}$ siècle, la persuasion nécessite tout d'abord une meilleure compréhension du monde environnant, ce qui permet au texte de gagner en intelligence et en rationalité. Le mouvement de bascule faisant naître le texte à dominante explicative s'effectue justement à la fin du XIII ${ }^{e}$ siècle et s'accentue tout au long du moyen français. À la fin du XIII siècle, ce sont les encyclopédistes qui abordent presque tous les sujets relatifs au monde physique: la cosmographie, la création et la procréation, les animaux, l'histoire et la géographie, la géologie, l'astronomie, la médecine, la diététique, etc. Ces textes à vocation essentiellement référentielle résument l'activité intellectuelle d'une époque et fournissent le catalogue presque complet des idées admises, produites et circulant, à l'origine, à l'intérieur d'une communauté plus restreinte, et s'ouvrant progressivement vers l'extérieur grâce à une activité de diffusion qui s'inscrit dans un ensemble comprenant traduction, résumé, reformulation et réécriture de textes sources en direction de ce nouveau lectorat.

Le discours des encyclopédistes se veut avant tout explicatif. Cependant, il est important de souligner que le type explicatif-informatif se forge au contact de tous les autres et assimile donc des traditions d'écriture correspondant à des discours à dominante narrative, descriptive ou dialogale. Dans son ouvrage Science médiévale 
et vérité: étude linguistique de l'expression du vrai dans le discours scientifique en langue vulgaire, Silvi résume bien cette rencontre des genres et donc de leurs modèles séquentiels dominants dans le discours de vulgarisation scientifique:

Qu'on ne s'étonne donc pas de trouver des rapprochements, toujours ponctuels et rapides, avec le roman courtois, la chanson de geste, le récit de l'historien ou celui du marin, l'œuvre de Chrétien de Troyes, le texte juridique ou bien encore le sermon. Il nous a paru intéressant de souligner ce que le discours scientifique pouvait avoir de commun avec tous ceux-là, mais surtout de montrer comment il utilise à des fins qui lui sont propres des procédés tellement traditionnels qu'on en finissait par penser qu'ils ne pouvaient fonctionner autrement et qu'on en arrivait même à ne plus les voir. Sous nos yeux, le cliché se réactive, le sens qu'on croyait à jamais perdu est réactualisé et l'image, morte, s'anime à nouveau. Les procédés de crédibilisation de l'énoncé sont multiples, mais ils ont toujours, pour celui qui est un peu familier avec la littérature médiévale, un air de déjà-vu.

(Silvi, 2003: 21)

Cette «réactivation des clichés», pour reprendre les termes utilisés par Silvi, correspond au fait que les auteurs qui mettent en langue vulgaire un savoir spécifique afin de l'expliquer, reprennent, dans un premier temps, les formes d'organisation et les stratégies discursives pratiquées dans les textes à dominante narrative. En effet, le public laïc a déjà une certaine culture littéraire qui détermine son horizon d'attente et qui est essentiellement religieuse, historique, narrative, quels que soient le genre littéraire (roman, théâtre, dit...) et le mode de transmission (oral ou écrit) considérés. La vulgarisation du savoir, au double sens de «mise en langue vulgaire» et de «mise à la portée d'un public non savant», pose la question de l'adéquation de la forme littéraire au contenu à transmettre et à la visée didactique qui l'oriente; ce questionnement renvoie aux cadres de l'argumentation définis par Perelman et Olbrechts-Tyteca qui ont souligné l'importance de la «connaissance de l'auditoire [qui] ne se conçoit pas indépendamment de celle des moyens susceptibles d'agir sur lui» (Perelman et Olbrechts-Tyteca, 1976: 30). L'adaptation à un public culturellement différent du savant produit des discours caractérisés par une hétérogénéité profonde car reprenant des schémas séquentiels traditionnels tout en les adaptant aux nouvelles contraintes de l'acte de langage mis au premier plan, celui d'expliquer un savoir et ses composantes. Étant donné cet appui sur des stratégies discursives existantes, et plus particulièrement le recours aux modèles séquentiels narratif et descriptif $^{9}$, la «réactivation des clichés» inclut-elle le maintien de la digression? La digression est-elle compatible avec le registre didactique, extrêmement structuré et rigoureusement organisé?

9. «Agrémenter le texte essentiellement descriptif (apparemment homogène au contenu scientifique) de pauses narratives, plus adéquates au public visé et donc au but poursuivi (faire comprendre le monde créé et aimer son Créateur) relève d'un savoir-faire, d'un savoir-écrire que met en œuvre au XIII ${ }^{e}$ siècle chacun des auteurs de ces encyclopédies en langue vulgaire» (Connochie-Bourgne, 2006: 118). 
Nous tenterons d'apporter quelques éléments de réponse à ces questions en nous intéressant plus particulièrement aux marques d'inscription du digressif dans l'explicatif et à l'objectif de cette stratégie mise en place dans des ouvrages dont la fonction est de dispenser des enseignements, de transmettre un savoir ou une morale.

\subsection{Digression, illustration et exemplum: parler d'autre chose ou de la même chose?}

Comme nous l'avons déjà souligné dans l'introduction à cette étude, il existe une parenté entre la digression et l'exemplum. Nous proposons d'introduire dans notre réflexion l'argumentation par l'illustration qui, au même titre que l'argumentation par l'exemple, fait partie des «liaisons qui fondent le réel par le recours au cas particulier» (Perelman et Olbrechts-Tyteca, 1976: 471). Dans les trois cas, nous avons affaire à une rupture du discours, à une rupture de la chronologie du texte, à une insertion comparable à des parenthèses. Cependant, contrairement à la digression, l'exemplum et l'illustration se situent dans l'axe logique de l'argumentation dont ils doivent expliciter la portée concrète. Tandis que l'exemple permet une généralisation, «l'illustration a pour rôle de renforcer l'adhésion à une règle connue et admise, en fournissant des cas particuliers qui éclairent l'énoncé général [...]» (Perelman et Olbrechts-Tyteca, 1976: 481). La différence entre la digression d'un côté et l'exemplum et l'illustration de l'autre réside dans la force et la pertinence du lien que ces insertions entretiennent avec le cadre dans lequel elles s'inscrivent. La digression implique un éloignement par rapport au sujet annoncé; elle repose sur une opposition entre un cadre nécessairement premier et un décrochage par définition secondaire et nouveau. L'exemplum, quant à lui, se greffe sur le schéma explicatif/argumentatif mis en place dans le cadre afin de le compléter. Il représente l'argument concret, celui qui sollicite l'imagination du lecteur. L'exemplum est bien un argument parmi d'autres. Cet argument se présente sous forme de micro-séquences narratives au service de la persuasion. Ces exemples pédagogiques visent à prouver la vérité des affirmations avancées par le maitre. Ainsi, pour expliquer à son élève par exemple la corporéité des quatre éléments, le philosophe Timéo ${ }^{10}$ rapporte-t-il l'histoire suivante:

[1] Uns paysans aloit coeullir en .I. desert graine et portoit avec luy une grant aucelle, grosse et longue, parfonde et estroite a l'entree par le bouque et estoit plain d'iaue [...]. Une fois avint que chus ot par .XV. jours beu son yaue si son vaissel fut moult apetichiés [...].

(Placides et Timéo ou li secrés as philosophes, p. 34-35)"

10. Placides et Timéo, dernier quart du XIII ${ }^{\mathrm{e}}$ siècle. Il s'agit d'une encyclopédie en prose anonyme, sous forme d'un dialogue entre le philosophe Timéo et son disciple, le prince Placides. Les questions et réponses forment une succession de traités sur les sujets les plus divers: la métaphysique, la reproduction, la météorologie, l'astrologie, etc. Ce texte se présente à la fois comme une encyclopédie dialoguée, un miroir des princes et un petit recueil de récits exemplaires.

11. Sauf mention contraire, les références complètes des éditions citées et des traductions en français moderne sont données dans la bibliographie de fin d'article. 
'Un paysan allait cueillir des graines dans un désert. Il apportait une grande cruche qui était lourde et haute, avec un bec profond et étroit. La cruche était pleine d'eau [...]. Mais une fois le paysan avait bu de son eau pendant quinze jours et le niveau d'eau de la cruche avait beaucoup diminué [...].'

(notre trad.)

Suite :

Il advint aussi qu'un corbeau, fuyant des oiseaux de proie, se réfugia précisément dans ce «désert» dépourvu de tout point d'eau; il advint encore qu'il vit la cruche à côté du paysan endormi, mais il comprit qu'il ne pouvait ni atteindre le niveau de l'eau avec son bec ni la renverser tant la cruche était lourde; il remplit donc de cailloux le récipient, fait monter le niveau de l'eau et étanche sa soif. En bon éducateur du prince, Timéo conclut: «et par ce monstra il que c'est voirs que vous di au commencement que mieux vaut sens que force». Mais la fable a illustré surtout la notion scientifique: «et si monstra semblant que il seut que l'iaue eüst corps». La sagacité du corbeau est un défi à l'intelligence de l'élève.

(exemple présenté par Connochie-Bourgne, 2006: 129-130)

Dans le discours scientifique, l'exemplum a le statut d'une parole d'autorité. Il est «d'autant plus rentable qu'il permet, comme c'est le cas lorsqu'il appartient à une compilation, de répéter la même chose, mais selon des modalités différentes: les personnages, les lieux, les aventures changent, mais c'est toujours la même vérité qui est dite» (Silvi, 2003: 416). Ce constat vaut également pour les traités didactiques. En fonction de la nature de l'enseignement dispensé, l'exemplum ou l'illustration permettent de convaincre le lecteur sur les plans scientifique et/ou moral $^{12}$. Dans l'extrait suivant du Mesnagier de Paris (1393), c'est un exemple à valeur illustrative et visée moralisatrice qui complète et appuie l'argumentation/explication du discours-cadre:

[2] $\{17\}[\ldots]$ Chiere seur, vueilliez de par moy sur ce savoir que quiconques, soit homme ou femme, qui veuille a droit ses pechiez confesser au sauvement de l'ame de lui ou d'elle, il doit savoir que trois choses sont necessaires; c'estassavoir; contriction, confession et satisfaction.

\{18\} Et doit il ou elle savoir que contriction requiert douleur de cuer en grans gemissemens et repentences, et couvient que en grant contriction et treshumblement le pecheur requiert pardon [...]. Et sache le pecheur que sans contriction sa priere ne vault riens, puis qu'il ait sa pensee en son cuer ailleurs. Et, chiere suer, vous en pouez prendre exemple par ung a qui l'en promist donner un cheval, pour dire une paternostre, mais qu'il ne pensast autrepart. Et en disant la paternostre il se pensa se celui qui lui donnoit le cheval lui laisseroit la selle; et ainsi le malheureux

12. «L'exemplum à visée scientifique est une histoire dans laquelle la connaissance l'emporte sur la visée moralisatrice, celle-ci étant accessoire et secondaire» (Silvi, 2003: 424). 
perdit tout. Ainsi est il de cellui qui deprie Nostre Seigneur et ne pense point a sa priere ne a cellui qu'il deprie [...].

(Le mesnagier de Paris, I, iii, «Enseignement catéchistique et moral»)

‘ $\{17\}[\ldots]$ Chère amie, apprenez de moi à ce sujet que quiconque, homme ou femme, souhaite confesser convenablement ses péchés pour sauver son âme, doit savoir que trois choses sont nécessaires: contrition, confession et satisfaction.

$\{18\}$ Il ou elle doit savoir que la contrition requiert une douleur au cœur qui se manifeste par de grands gémissements et regrets; il convient que le pécheur, fortement contrit et humble demande pardon [...]. La prière d'un pécheur sans contrition ne vaut rien, qu'il le sache, à partir du moment où, en lui-même, sa pensée est ailleurs. Chère amie, écoutez à ce propos l'exemple de celui à qui on promit de donner un cheval en échange d'un Notre Père, à condition qu'il ne pense à rien d'autre pendant la récitation. Tout en s'exécutant, l'homme se demanda si en plus du cheval on lui donnerait aussi la selle; de cette manière le malheureux perdit tout. Ainsi en va-t-il de celui qui implore Notre-Seigneur sans se concentrer et sans penser à Celui à qui elle s'adresse [...].'

Vers 1393, un bourgeois de Paris, riche et vieillissant, écrit pour sa très jeune épouse un ouvrage qui mêle l'instruction religieuse et morale, des conseils d'économie ménagère et des recettes de cuisine très nombreuses. Dans cet ouvrage, Le mesnagier de Paris, le mari esquisse les contours d'un idéal féminin - celui d'une femme mariée observant strictement toutes les règles morales, religieuses et sociales. Cet idéal bourgeois est peint dans les chapitres d'enseignement moral, conjugal et catéchistique, mais encore à travers les nombreux exempla et micro-récits à valeur illustrative. Dans l'exemple [2], extrait du troisième article de la première leçon, l'auteur du Mesnagier explicite la portée concrète de son discours injonctif, centré sur la sincérité de la confession, par une insertion narrative. Cette dernière est annoncée par un métaterme - exemple - qui marque l'entrée dans l'espace discursif occupé par le micro-récit. Il est à noter que chacune des micro-propositions du fragment $\{18\}$ commence par le connecteur $e t$, marque caractéristique de la rhétorique didactique qui se distingue par son goût pour l'énumération. La reprise de ce connecteur signale qu'aussi bien les fragments argumentatifs ou explicatifs enchâssés dans le discours injonctif que la narration s'inscrivent sur le même axe logique de l'argumentation. La présence de cet axe logique conditionne la cohérence du dispositif argumentatif. L'enseignement dispensé par le mari est organisé dans le sens du développement de l'hyperthème «trois choses» (sont nécessaires pour se confesser convenablement). À chacun des sous-thèmes («contrition, confession et satisfaction») correspond un passage argumentatif-explicatif rédigé dans une modalité instructionnelle et qui, dans le cas de la contrition, est complété par un exemple. L'espace discursif occupé par ce dernier est délimité par deux énoncés qui sonnent, le premier, comme un avertissement ( EEt sache le pecheur que sans contriction sa priere ne vault riens, puis qu'il ait sa pensee en son cuer ailleurs») et, le second, comme le récapitulatif («Ainsi est il de cellui qui deprie Nostre Seigneur et ne pense point a sa priere ne a cellui qu'il deprie») 
d'une leçon à retenir. Dans le connecteur ainsi du second énoncé se combinent les valeurs de récapitulation et d'illustration. L'exemple donné a donc le statut d'une illustration qui éclaire l'argumentation générale par un cas particulier. Le connecteur ainsi prolonge le mouvement textuel mis en place par la combinaison des connecteurs «et ainsi», située à l'ouverture de la proposition qui clôt le récit proprement dit («et ainsi le malheureux perdit tout»), et fonctionne comme un relais entre l'insertion narrative et le discours-cadre. L'effet de symétrie créé par les reprises aux niveaux du lexique et du fonctionnement syntaxique (structures de négation) dans les énoncés qui délimitent l'illustration, contribue à la cohésion de l'ensemble formé par le cadre et le micro-récit.

\section{2. À propos de la digression...}

La recherche systématique de l'ordre logique qui caractérise le discours didactique du Moyen Âge devrait faire obstacle à la digression. On constate cependant pour la période du $\mathrm{XII}^{\mathrm{e}}$ au $\mathrm{XV}^{\mathrm{e}}$ siècle un emploi récurrent qui «témoigne d'une volonté sommative, avouée, voire revendiquée, par les auteurs d'encyclopédies, de traités didactiques, de récits de voyages, de chroniques, plus masquée dans les œuvres de fiction» (Connochie-Bourgne, 2005: 8). Sous quelle forme cette volonté se manifeste-t-elle dans le discours à intention explicative/argumentative? Quels marqueurs permettent de délimiter les passages digressifs? En effet, la définition du digressif met en évidence l'importance du marquage. Cette importance se conçoit: interrompre un énoncé, le réorienter puis renouer avec le propos primitif sont des opérations cognitivement coûteuses, nécessitant qu'on oppose au déroulement organisé du discours des «obstacles» dotés d'une capacité suffisante à agir et à inscrire dans le discours des strates entretenant des liens hiérarchiques complexes.

Les manifestations du digressif dans des séquences à intention explicative/ argumentative peuvent être implicites ou explicites.

\subsubsection{Délimitations implicites des passages digressifs}

Selon Ueltschi, «dans la littérature didactique, beaucoup de digressions sont implicites, se présentent comme une déviation involontaire du propos principal» (Ueltschi, 2005 : 394). L'attribution du qualificatif «involontaire» à ces déviations renvoie à l'idée selon laquelle «la digression entretient avec son sujet principal un lien de nature» (Milhe Poutingon, 2012: 19). Ce lien certain avec le sujet s'impose afin de garantir la cohérence de l'ensemble. Il relève de ce que Bellert (1970) appelle les «quasi-implications». Ces dernières doivent être comprises comme des «règles générales de raisonnement» permettant de découvrir les relations entre des états de choses décrits par deux énoncés successifs via des connaissances du monde associées. La digression profite ainsi des possibilités offertes par le discours via le raisonnement afin de produire des déviations par des changements d'axe logique installant une nouvelle perspective dans le récit. Celle-ci peut être déclenchée par un effet de symétrie, comme dans l'extrait suivant du discours didactique du Mesnagier de Paris: 
[3] $\{1\}$ Le.Vii. Article de la premiere distinction doit demonstrer que vous devez estre curieuse et songneuse de la personne de vostre mary. Sur quoy, belle suer, se vous avez autre mary après moy, sachiez que vous devez moult penser de sa personne. [...] Et vous pry que vous le tenez nectement de linge, car en vos en est. Et pour ce que aux hommes est la cure et soing des besongnes de dehors, et en doivent les mariz songner, aller, venir et racourir deça et dela par pluyes, par vens, par neges, par gresles, une foiz moullié, une foiz sec, une foiz suant, autresfoiz tramblant, mal peu, mal hebergié, mal chaussié, mal couchié - et tout ne luy fait de mal pour a ce qu'il est reconforté de l'esperance qu'il a aux cures que la femme prendra de luy a son retour [...] : d'estre deschaux a bon feu, d'estre lavé les piez, avoir chausses et soullez fraiz [...]. Et l'endemain robes-linges et vestemens nouveaulx.

(Le mesnagier de Paris, I, vii, «Prendre soin du mari et de la maison»)

' $\{1\}$ Le septième article de la première distinction a pour objet de vous enseigner à être attentive et prévenante à l'égard de votre mari. Sachez à ce sujet, belle amie, que si vous avez un autre mari après moi, vous devez très bien vous occuper de sa personne. [...] Je vous prie de le pourvoir toujours de linge impeccable, car c'est là votre domaine. Les occupations et les travaux de l'extérieur sont pour les hommes. Les maris doivent y pourvoir, aller, venir, courir çà et là, qu'il pleuve, vente, neige ou grêle, tantôt mouillés, tantôt secs, tantôt en sueur ou tremblants de froid, mal nourris, mal logés, mal chaussés, mal couchés. - Tout cela ne lui fait rien parce qu'il est réconforté en pensant aux soins que sa femme prendra de lui à son retour [...] : le déchausser auprès d'un bon feu, lui laver les pieds, lui donner des chausses et des souliers propres [...]. Et le lendemain lui préparer une chemise et des habits nouveaux.'

Tandis que le discours-cadre est centré sur les soins que la jeune épouse doit prodiguer à son mari, la déviation produit, par effet de symétrie ou plutôt de rééquilibrage, un discours consacré aux devoirs des hommes envers leurs épouses. Le développement concerné se situe bien dans l'axe thématique général - l'organisation du ménage et la présentation du mariage comme cadre de vie idéal - mais il est digressif par rapport au projet particulier du Mesnagier qui consiste à donner un enseignement général à la jeune épouse. L'espace discursif occupé par la digression est connecté au cadre-mère par la conjonction et qui matérialise l'existence d'un lien sans que ce dernier soit explicité. Le fragment discursif ainsi introduit dévie du développement principal par la mise en place d'une perspective d'opposition entre les femmes et les hommes, doublée par l'opposition entre intérieur et extérieur. Le décrochage par rapport au plan principal se manifeste également au niveau énonciatif par un «effacement» de l'allocutrice qui n'est plus que représentée par le syntagme nominal «la femme». L'établissement d'une relation de congruence entre les aspects opposés reste possible grâce à la constante thématique du devoir de réalisation de certaines occupations ou tâches («vous devez estre curieuse et songneuse [...]»; «et en doivent les maris songner»).

Le rattrapage énonciatif et donc le retour à l'axe principal de la démonstration ont lieu avec le passage au paragraphe suivant qui remet en place la simulation de dialogue caractéristique de l'ouvrage («Certes, belle seur, [...]», I, vii, § 2). Rappelons 
à cette occasion que dans les manuscrits médiévaux le passage à un nouveau fragment textuel n'est souvent matérialisé que par un changement de la taille des caractères, des lettrines ou un changement de couleur. La notion de paragraphe n'étant pas encore établie, le texte était souvent écrit linéairement, sans sauts de lignes entre les différentes parties d'un exposé ou d'un récit. Les rubriques (livres, chapitres, sections...) étaient séparées par des phrases, souvent colorées. En tant qu'information apparente intentionnelle d'un texte manuscrit, la rubrication (réalisation de rubriques ou de passages à l'encre rouge) faisait ressortir les articulations principales du texte.

La comparaison constitue un autre embrayeur de la digression. Elle est attestée dans l'exemple suivant, extrait des commentaires de la traduction française de l'Éthique à Nicomaque d'Aristote réalisée par Nicole Oresme (vers 1370). Félicité humaine, félicité divine: tel est le parcours d'Aristote qu'Oresme suit dans sa traduction et ses gloses. Oresme reprend la définition aristotélicienne du bonheur tout en s'interrogeant sur le lien entre le bonheur et la richesse. Cette réflexion sert de cadre à une déviation de l'axe principal, déclenchée par une comparaison tellement développée qu'on perd de vue qu'il ne s'agit que d'une comparaison: elle devient en fait le sujet principal du discours:

[4] $\{10\}$ Il procede yci a la seconde question principal de cest chapitre, a savoir mon se fortune puet oster ou muer felicité aprés la mort. [...]

$\{13\}$ Et pour ceste cause, quant un home avoit esté bon et avoit eüe bonne fortune toute sa vie, Solon disoit que adonques aprés sa mort l'en povoit bien dire que il avoit esté beneuré.

$\{14\}$ [...] Et selon l'opinion de Solon il y convient .II. choses, c'est assavoir, que il soit bon et que il ait bonne fortune; et se il li faut une de ces choses, il est maleureus. $\{15\}$ Plinius dit que c'est une petite beste comme une lesarde et que elle vit de l'air non pas pur et senz mangier, et tout son sanc est ou cuer et es yex qui sont gros et ne les clost onques. Et se mue et varie en toutes telles couleurs comme ont les choses que elle resgarde longuement, excepté .II. couleurs, rouge et blanche. Ceci dit Plinius. Et la cause puet estre, si comme je cuide, car quant ceste beste adresce son voiement a une couleur, ceste couleur multiplie son espece par les yex de la beste en son corps, lequel se trait a nature de air et est transparent dedenz. Et nous voions bien aucunes choses par quoy nous les povons croire, ja soit ce que ce ne soit pas du tout semblable. Car quant le soleil luist encontre .I. drap vert ou par une verriere verte, les choses opposites semblent estre vertes. Mais de ce que Plinius en excepte deux couleurs puet estre que celle beste est rouge de sa propre nature et appert rouge toutes foiz que elle ne regarde longuement et fort a grant entencion une autre couleur. Et pour ce ne se varie elle pas a regarder couleur rouge. Et blanche couleur est comme lumiere. Et veons en l'example devant mis que les choses ne muent pas en apparance leur couleur pour ce se le soleil luist par my une verriere blanche ou contre une paroy blanche. A retourner a propos ${ }^{13}$, le cameleon mue et varie souvent

13. C'est nous qui soulignons. 
ses couleurs pour les choses dehors; et ainsi seroit il d'un homme se sa felicité estoit variee et muee pour les fortunes, car il viennent dehors.

(DMF: N. Oresme, Le livre de Ethiques d'Aristote [commentaires] [1370], New York, G. E. Stechert, 1940, p. 133)

' $\{10\}$ Il passe maintenant à la seconde question principale de ce chapitre qui consiste à savoir si la richesse peut provoquer la disparition ou un changement de la félicité après la mort. [...]

$\{13\}$ Et pour cette raison, selon Solon, on pouvait bien dire d'un homme bon et bien fortuné pendant sa vie qu'il avait été heureux après sa mort.

\{14\} [...] Et pour cela, selon Solon, il faut que deux choses soient réunies: il faut qu'il soit bon et qu'il dispose d'une bonne fortune; si l'une de ces deux choses lui fait défaut, il est malheureux.

\{15\} Plinius dit que c'est une petite bête comme un lézard [...] et tout son sang se situe dans son cœur et dans ses yeux qu'il ne ferme jamais. Cette bête modifie sa couleur en s'adaptant à celle des objets qu'elle regarde longuement, à l'exception de deux couleurs: le rouge et le blanc. [...] Et la raison peut être, comme je le pense, que quand le regard de cette bête tombe sur une couleur, l'image de cette couleur se répand par les yeux dans l'intérieur du corps qui ressemble à l'air par sa transparence. [...] Quand le soleil brille contre un tissu vert ou un vitrail vert, les choses qui se trouvent en face semblent être vertes. Mais puisque Plinius en excepte deux couleurs, il est possible que cette bête soit rouge de nature et qu'elle conserve la couleur rouge à chaque fois qu'elle ne regarde pas de façon assez longue et assez intense une autre couleur. Et pour cette raison, elle ne change pas de couleur non plus quand son regard tombe sur du rouge. Et la couleur blanche est comme la lumière. Et par cet exemple nous comprenons que les choses ne changent pas en apparence de couleur parce que la lumière du soleil traverse un vitrail blanc ou frappe contre un mur blanc. Pour retourner à notre propos et donc au caméléon, il modifie et change souvent ses couleurs sous l'influence des objets extérieurs; et ainsi serait-il d'un homme si sa félicité était modifiée par les richesses, car ces dernières viennent de l'extérieur.'

(notre trad.)

Dans cet exemple, la digression prend la forme d'une séquence enchâssée complexe dans laquelle s'emboîtent l'explication, l'argumentation et la description qui, quant à elles, résultent de deux types de réflexion: une réflexion personnelle, subjective («la cause puet estre, si comme je cuide»), et une réflexion neutre, objective, qui correspond à la voix de la doxa ou au discours de véridiction. Ce deuxième type de réflexion est représenté par Plinius qui fonctionne comme argument d'autorité inaugurant le paragraphe $\{15\}$ dans lequel s'inscrit la digression. C'est la comparaison attribuée à Plinius et selon laquelle la félicité «est une petite beste comme une lesarde» qui fait basculer le discours tout en le transformant en un «lieu d'une subjectivité énonciative performative et argumentative» (Ueltschi, 2005 : 404). Le statut du fragment $\{15\}$ correspond à celui d'un passage de second plan à dominante descriptive, 
caractérisé par une organisation interne qui le rend en quelque sorte indépendant $\mathrm{du}$ contexte dans lequel il s'insère ${ }^{14}$. Cette autonomisation justifie l'indication du retour à l'axe logique principal («A retourner a propos»). La continuité discursive - et donc la cohésion - entre le cadre-mère et la déviation est maintenue par le biais du lien logique de causalité se manifestant dans la texture du passage digressif ( «Et la cause puet estre»; «car quant ceste beste $[. .]$.$» ; « Car quant le soleil luist [...]») et dans$ le contexte d'insertion («et ainsi seroit il d'un homme se sa felicité estoit variee et muee pour les fortunes, car il viennent debors»). Quant à la cohérence de cette insertion, elle est garantie par le fait que le texte dont elle fait partie est une traduction commentée révélant la pensée scientifique d'un auteur-traducteur qui inscrit la relation entre les faits dénotés dans le cadre d'un raisonnement par analogie. L'éloignement par rapport au sujet principal est ainsi appuyé par le fait que le thème (la félicité dans sa relation avec la richesse) et le phore ${ }^{15}$ (le lézard) appartiennent à des domaines différents (phore du domaine sensible, thème du domaine spirituel).

D'autres déclencheurs implicites de digressions sont l'articulation entre le général et le particulier ${ }^{16}$ ainsi que le glissement métonymique. Nous avons relevé un exemple pour cette dernière catégorie de déclencheurs dans un ouvrage du XVII ${ }^{e}$ siècle, période pendant laquelle «les écrivains recherchent la digression pour ses fonctions ornementales, heuristiques [...] tout en conservant peu ou prou des réflexes scolastiques qui les poussent à rejeter le hors-sujet» (Milhe Poutingon, 2012: 45). Il s'agit du Théâtre d'agriculture et mesnage des champs d'Olivier de Serres qui représente une somme agronomique sur toutes les productions de produits vivriers et leurs utilisations. Appuyé sur les connaissances du Moyen Âge et de la Renaissance, l'auteur y développe la philosophie de la gestion dite «en bon père de famille» avec l'objectif de préserver le bon fonctionnement et l'avenir de l'exploitation:

[5] La cuisine donques, à telle cause sera posee au premier estage de la maison, au plan $\&$ prés de vostre salle, de laquelle entrerés dans vostre chambre: par ainsi ceux qui sont dans la cuisine par l'approche de la salle \& de la chambre, où estes souvent, s'en treuvent contrerollés \& reprimees les paresses, crieries, blasphemes, larcins des serviteurs \& servantes. Mesme la nuict, quand les servantes, sous petexte de fourbir leur vaisselle, faire leur buee $\&$ autres ordinaires mesnageries, demeurent bien tard dans la cuisine: mais vous sentans prés d'elles, n'auront lors moien de ribler avec les serviteurs, à l'aise $\&$ sans crainte: ainsi que cela est facile $\&$ commun en la cuisine

14. «[...] la progression thématique s'y exerce de façon interne, pourrait-on dire, avec le développement de progressions spécifiques et, du point de vue syntaxique, un nouveau statut pour l'ordre direct SVX. Toutes ces propriétés se combinent pour aboutir à la création d'un type de texte, identifié et marqué comme tel. Il conviendrait d'ailleurs de déterminer jusqu'à quel point ce sont les textes argumentatifs et informatifs, dans lesquels cette autonomie du second plan semble émerger dès le XIV ${ }^{e}$ siècle, qui ont pu influencer les productions littéraires» (Combettes, 2012a: 8-9).

15. Voir Perelman et Olbrechts-Tyteca, 1976: 501.

16. Nous renvoyons à l'article de Ueltschi (2005) consacré au Mesnagier de Paris, dans lequel l'articulation entre le général et le particulier est un déclencheur implicite très fréquent de la digression. 
basse, le maistre \& la maistresse estans retirés en leur chambre en haut, loin d'elles, $\&$ laissees comme en pleine liberté.

(O. de Serres, Le théâtre d'agriculture [1600], p. 22)

'Pour cette raison donc la cuisine sera installée au premier étage de la maison, à proximité de votre salon qui vous donnera accès à votre chambre: c'est grâce à cette proximité par rapport à votre salon et votre chambre que ceux qui sont dans la cuisine seront contrôlés et empêchés d'être paresseux, de crier, blasphémer et de commettre des vols. Même la nuit, quand les servantes, sous prétexte de nettoyer la vaisselle, faire la lessive et d'autres tâches ménagères, restent longtemps dans la cuisine. En sentant votre proximité, elles ne se livreront pas facilement et sans crainte à la débauche avec les serviteurs. Cela est plus facile et largement répandu dans la cuisine au rez-de-chaussée [...].'

(notre trad.)

Au chapitre $\mathrm{V}$ du premier livre du Théâtre d'agriculture, l'auteur dessine le plan de la maison champêtre. La justification de la situation de la cuisine fait dévier l'axe logique du discours : la préoccupation des maitres de la maison, à savoir le contrôle des serveurs et servantes, prend le dessus. Le thème du discours se déplace ainsi de la maison vers ses habitants.

\subsubsection{Délimitations explicites de la digression}

Pour Sabry, la digression possède des signes formels et se manifeste en tant que telle: «Toute digression contient un discours plus ou moins bref sur elle-même: elle se nomme, se justifie, s'accuse d'inutilité, de longueur, d'incorrigibilité, de manque d'à-propos ou proclame sa nécessité, son apport [...]» (Sabry, 1992: 93). En principe, une digression est bornée, en amont comme en aval, par des formules que Fouquelin, dans sa Rhétorique française (1557), nomme "préface» et "clausule» ${ }^{17}$. L'importance des marqueurs se conçoit car, comme nous l'avons déjà constaté, l'interruption d'un discours, sa réorientation et le retour au propos primitif, sont des opérations cognitivement coûteuses. Milhe Poutingon précise que «plus des informations sont reliées entre elles dans le modèle mental, moins elles ont besoin d'être connectées en discours. À l'inverse, moins elles sont reliées plus la nécessité de les connecter est grande» (Milhe Poutingon, 2012: 166).

Les signaux explicites de l'inscription du digressif dans l'explicatif-argumentatif relèvent essentiellement de trois catégories: les marqueurs d'insertion, les signes de ponctuation et signes typographiques, les «béquilles argumentatives» (Ueltschi, 2005: 396).

17. «Digression est une interruption de propos et changement en un autre, non étrange du tout: laquelle est quelquefois un peu plus longue, quelquefois plus courte. La Digression plus longue est volontiers prémunie d'une petite et brève préface, de peur que inconsidérément et sans y penser nous semblions aller du coq à l'âne: et à la fin d'icelle on a de coutume mettre une petite clausule signifiant la retraite et rentrée au propos, duquel on était sorti» (A. Fouquelin, La rbétorique française, in Traités de poétique et de rbétorique de la Renaissance, F. Goyet (éd.), Paris, Librairie générale française, 1990, p. 420). 


\subsubsection{Les marqueurs d'insertion}

Ces marqueurs définissent le cadre d'insertion du détour dans le propos primitif. Ils se manifestent sous forme de locutions prépositionnelles (par especial [«en particulier», «surtout»], d'ailleurs) ou de paradigmes métadiscursifs désignant le cadre d'insertion comme un "propos» ou un «sujet» (pour reprendre le propos; pour retourner à mon principal sujet). La digression se marque surtout après coup, peut-être parce que la borne située à l'entrée exprime plus fortement l'idée d'interruption alors que la formule de clôture signale le retour dans le discours-cadre. L'exemple [4] témoigne de ce type de fonctionnement: la déviation discursive se met en place de manière implicite (par une comparaison), la clôture est signalée explicitement par la formule «a retourner a propos».

Mais on relève aussi des exemples, plus rares, où la digression n'est bornée qu'en amont, comme en témoigne l'extrait suivant du Recueil des plus célèbres astrologues de Simon de Phares:

[6] \{3686\} Cycrops, premier roy de Athenes. Cestui fut astrologien, jaçoit qu'il institua plusieurs supersticions en Grece, par especial en ce qu'il sacriffia premier à Jupiter ung beuf. Lequel beuf en son temps yssit d'un fleuve et monta en l'air le jour de la feste Serapis et après descendit oudit fleuve et depuis ne apparut, pour laquelle chose icelui Cicrops ordonna que on offrist le beuf plus tost que autres bestes. De cestui parle Eusebe ou quart de son euvre. Cestui Cycrops fonda Athenes [...].

(DMF: S. de Phares, Recueil des plus celebres astrologues [1494], Paris, H. Champion, 1929, p. 27)

'\{3686\} Cécrops, premier roi d'Athènes. Il était astrologue, bien qu'il fût à l'origine de plusieurs superstitions en Grèce, surtout parce qu'il était le premier à sacrifier un bouf à Jupiter. Ce bøuf sortit d'un fleuve et monta dans l'air le jour de la fête de Sérapis, après il redescendit dans ce même fleuve et depuis il ne réapparut plus. Pour cette raison, Cécrops ordonna de sacrifier un bœuf plutôt qu'un autre animal. Eusèbe parle de lui dans une partie de son œuvre. Ce Cécrops fonda Athènes [...].' (notre trad.)

Dans cet exemple, le retour à l'axe principal - Cécrops-astrologue et premier roi d'Athènes - est signalé par le décrochage temporel («De cestui parle Eusebe») ainsi que par la reprise de l'une des caractéristiques du personnage, annoncée au début du fragment \{3686\} («Cycrops, premier roy de Athenes [...] Cestui Cycrops fonda Athenes»).

Dans le cas de doubles marquages, les signaux que sont «préfaces» et «clausules» s'articulent souvent sur toutes sortes de connecteurs servant à joindre le cadre: «Mais, quoy qu'il en soit, pour revenir à mon premier propos [...]» (H. Estienne, Deux dialogues du nouveau langage françois italianizé [1578] ; exemple cité par Milhe Poutingon, 2012: 167). La nécessité de joindre est telle que, dans certains cas, ces connecteurs font seuls office de marques: 
[7] Ne se trouvera donc estrange, si nous enseignons au pere de famille, que sur tout il entende et soit soigneux de sa bergerie plus que d'autre bestial, et face de sorte, que son tect aux ouailles soit bien equippé, et couvert en hyver, et son berger de bonne nature, bien comptable, et adroit à plusieurs choses honestes: car peu s'en trouvent aujourd'huy de cest estat (principalement pres des villes) qui par leur desidence et long repos, ne s'adonnent à quelque malice plustost qu'au bien ou profit de leurs maistres, en sorte que d'iceux, mal moriginez, journellement voyons venir plusieurs larcins, pilleries, voleries, et autres maux infiniz, au contraire les premiers bergers d'Egypte et d'ailleurs furent inventeurs de l'astrologie, art de medecine, musique, et plusieurs autres sciences liberales, je ne sçay si j'y dois adjouster l'art militaire, les principautez et gouvernement des Royaumes: parce que bien long temps ils parquoyent aux champs et vivoyent soubs la cabanne plusieurs annees, observants par loysir le cours des astres, les dispositions des saisons, et par long usage et experience marquans les felicitez et infelicitez du temps, en sorte que des anciens pasteurs sont venuz les gens de sçavoir en tesmoignage des Hieroglyphiques. Il faut donc en somme un grand soing et advis à choisir un bon berger.

(DMF: C. Estienne, L'agriculture et maison rustique, Paris, J. du Puis, 1564, p. 26)

'Il n'est donc pas surprenant si nous enseignons au père de famille de prendre soin surtout de sa bergerie [...] et de faire en sorte que l'abri des brebis soit bien équipé et couvert en hiver, et que son berger soit de bonne nature, rendant volontairement des comptes, bien disposé à l'égard de plusieurs choses honnêtes: car aujourd'hui on en trouve peu [...] qui ne se consacrent pas à des méchancetés plutôt qu'aux biens et aux intérêts de leurs maitres. Ces gens mal éduqués sont à l'origine de plusieurs crimes, pillages, vols [...], contrairement aux premiers bergers d'Égypte qui d'ailleurs ont inventé l'astrologie, l'art de la médecine, la musique et plusieurs autres sciences libérales. Je ne sais si je dois ajouter l'art militaire, les principautés et les gouvernements de royaume. Pendant bien longtemps ils s'installèrent aux champs et vivaient dans des cabanes durant plusieurs années, ils observèrent le cours des astres, le déroulement des saisons, et étaient capables suite à une longue expérience d'identifier les bonheurs et les malheurs d'une période. Les anciens bergers sont donc devenus des gens qui possèdent un savoir [...]. En somme, il faut donc choisir son berger avec beaucoup de soin et de réflexion.'

(notre trad.)

Cet extrait de l'un des premiers ouvrages en français consacré à l'art rural montre que les marqueurs explicites jouant le rôle de bornes se combinent avec d'autres signaux qui indiquent la déviation par rapport à l'axe principal, comme par exemple les discontinuités temporelle et spatiale ainsi que l'inscription d'une subjectivité énonciative performative («je ne sçay si [...]»). La cohésion de l'ensemble est maintenue par le biais du lien logique d'opposition, matérialisé par «au contraire».

Les topicalisateurs de type quant à, au regard de peuvent également jouer le rôle d'un marqueur de digression. Ils figurent de façon privilégiée dans les «préfaces» où ils signalent l'émergence d'un topique qui se situe en marge du discours-cadre: 
[8] Almansor, le grant astrologien de Castelle, fut en ce temps, selon aucuns. Cestui composa ung livre qui se commence: «Signorum disposicio», et calcula bien precisement les estoilles fixes qui de longtemps ne l'avoient esté, et est assavoir qu'ilz ont esté deux autres Almansors qui tous ont esté astrologiens. Mais cestui fut moult aprecié du roy d'Arragon. Au regard des autres, pour ce que je ne sçay leur temps, je les laisse, bien est il que plusieurs roys d'Arragon ont esté astrologiens. Yginus, .Xe. pappe, natif de Athenes, lequel fut en ce temps grant en toutes sciences $[\ldots]$.

(DMF: S. de Phares, Recueil des plus celebres astrologues [1494], Paris, H. Champion, 1929, p. 97)

'Almanzor, le grand astrologue de Castille, appartenait, selon certains, à cette période. Celui-ci composa un livre qui commence par "Signorum disposicio», et il calcula avec beaucoup de précision [la position] des étoiles fixes [...], mais il faut savoir qu'il y avait deux autres Almanzor qui étaient également astrologues. Mais l'Almanzor dont il est question a été très apprécié par le roi d'Aragon. Concernant les autres, dans la mesure où je ne sais pas à quelle période ils appartiennent, je les laisse de côté. Il est vrai que plusieurs rois d'Aragon ont été astrologues [...].' (notre trad.)

[9] Cornelius Celsus, et tous les anciens medecins tiennent que toutes morsures et esgratigneures, piqueures et bave des anmiaux, participent de quelque mauvaise qualité, toutefois les unes plus et les autres moins. [...]

Aucuns ne veulent excepter de ceste condition de morsure celle des hommes, affermans icelle participer de quelque venenosité, et principalement des rousseaux piquotés de marques tannées, noires et autre couleur, qu'ils ont partout leur corps, et encores plus s'ils sont en colere. Quant à ceux qui ne sont de tel temperament, on peut tenir leur morsure n'estre participante d'aucune venenosité à raison de leur salive, laquelle on voit par experience estant appliquée és petites ulceres, les guarir. Parqouy la difficulté qui vient de guarir la morsure qu'aura fait un homme non roux, vient à raison de la meurtrisseure qui se fait au moyen des dents, qui sont mouees et non trenchantes, lesquelles ne peuvent entrer dedans la chair sinon en escachant et contusant, comme se font les coups orbes et les playes faites avec des pierres et des bastons, ou autres semblables, lesquelles on voit estre plus difficiles à guarir que celles qui sont faites avec glaives trenchans. Et pour retourner à nostre propos, nous dirons qu'entre les bestes que nous avons dit estre les plus venimeuses, il s'en trouve peu qui soyent de tardive operation: mais elles font communément mourir soudainement.

(A. Paré, Animaux, monstres et prodiges [1573], p. 246-247)

'Cornelius Celsius et tous les anciens médecins soutiennent que toutes les morsures et égratignures, piqûres et baves d'animaux, sont nuisibles mais à des degrés variés. [...]

Pour certains, il en est de même pour les morsures faites par un homme. Ils affirment que celles-ci sont de nature vénéneuse, et principalement celles faites par les gens 
aux cheveux roux et dont le corps est garni de taches brunes, noires et d'autres couleurs, qui se multiplient quand ils sont en colère. Quant à ceux qui n'ont pas ce tempérament, on peut considérer que leur morsure n'est pas de nature vénéneuse en raison de leur salive [...] utilisée pour guérir. La difficulté qui consiste à soigner la morsure infligée par un homme non roux vient des marques faites par les dents, molles et non tranchantes, qui ne peuvent pas entrer dans la chair sans l'écraser et la meurtrir, comme c'est le cas des coups provoquant des contusions sans plaie ouverte et des plaies faites par des pierres et des bâtons [...] et qui sont plus difficiles à soigner que celles provoquées par des glaives tranchants. Et pour retourner à notre propos nous dirons que parmi les bêtes les plus venimeuses, il n'y en a que peu dont le venin agit tardivement [...].'

(notre trad.)

Dans ce dernier exemple, Paré, après avoir étudié les morsures et égratignures infligées par des animaux, introduit une réflexion sur les morsures faites par les hommes. Cette réflexion se présente sous forme d'une digression thématique, signalée par le changement de paragraphe. Ce type de digression est généré par ce qui au départ ne devait être qu'un commentaire ou une explication ponctuelle. Mais cette explication s'étoffe pour se transformer en développement digressif centré plus particulièrement sur l'opposition entre les morsures provoquées par les «rousseaux» et les «bommes non roux». Le topicalisateur quant à permet d'extraire le sous-thème «non roux» tout en le situant sur un axe dévié à la fois par rapport au sous-thème des «rousseaux» et au propos principal (les morsures infligées par les animaux). La cohésion de l'ensemble est garantie par le biais du lien logique d'opposition (les bêtes versus les hommes; les roux versus les non-roux).

\subsubsection{Les signaux de ponctuation et signaux typographiques}

Avec cette deuxième catégorie de marqueurs, nous nous situons du côté des opérateurs visuels participant à la segmentation graphique du texte. À titre d'exemple pour cette catégorie, on peut citer les mises en italique qui produisent un effet de contraste permettant de mettre en évidence des mots ou des passages afin de les distinguer du reste du texte. En tant que matérialisation de la stratégie digressive, cette mise en évidence est une signalisation explicite de la digression:

[10] Pour un prealable donques nostre père-de-famille sera averti de s'estudier a se rendre digne de sa charge, afin que sçachant bien commander ceux qu'il a sous soi, en puisse tirer l'obeïssance necessaire [...].

En cela imitant le General-d'armee, qui emploie aux fortifications, des pionniers, n'aians, comme beufs, autre valeur qu'en la force, sans esprit ni entendement. Sur ce sujet dit le Poëte:

Que son vers chante l'heur du bien-aisé rustique,

Dont l'bonneste maison semble une Republique.

(O. de Serres, Le théâtre d'agriculture [1600], p. 25) 
'Pour commencer, notre père de famille sera averti de s'appliquer pour se rendre digne de sa charge et savoir bien commander ceux qui sont sous ses ordres afin qu'il puisse obtenir l'obéissance nécessaire de ces derniers. [...] Il imite ainsi le général de l'armée qui emploie pour les travaux de fortification des ouvriers qui, comparables à des bœufs, n'ont d'autres valeurs que leur force et sont sans esprit et réflexion. Le Poète dit sur ce sujet: [...].'

(notre trad.)

Dans cet exemple, la digression est annoncée par une "préface» contenant le métaterme «sujet», et matérialisée par un opérateur visuel correspondant au marquage typographique ${ }^{18}$ du discours inséré.

Les parenthèses peuvent également être au service de la digression. En tant que ponctuant graphique, elles intègrent dans la continuité du texte ce qui aurait pu figurer dans une glose marginale. Dans l'extrait suivant du Mesnagier de Paris, on relève deux emplois successifs de ce signe de ponctuation:

[11] $\{12\}$ Pour oster tache de robe de soye, satin, camelot, drap de Damas ou autre: trempez et lavez la tache en verjus, et la tache s'en ira. Et mesmes se la robe est destaincte se revendra elle en sa couleur (ce que je ne croy pas). (Vertjus. Nota que ou temps que le vertjus nouvel se fait, l'en en doit prendre sans sel une fiole et la garder; car ce vault pour oster tache de robe et la mectre en sa couleur, et est tousjours bon, et nouvel et vieil.) (Le mesnagier de Paris, II, iii, «Les domestiques, le vin, le cheval»)

' $\{12\}$ Pour faire partir une tache sur un habit de soie, de satin, de camelot, de drap de Damas ou autres tissus précieux, il faut faire tremper puis laver la tache dans du verjus et elle partira. Et même si l'habit est décoloré, il retrouverait sa couleur initiale (mais à vrai dire j'en doute). (Verjus. Nota qu'il faut en prendre une fiole sans ajouter de sel au moment où le verjus nouveau se fait, et la conserver; le verjus, qu'il soit jeune ou vieux, est toujours un bon moyen pour enlever la tache d'un vêtement et lui faire reprendre sa couleur.)'

Les premières parenthèses incluent une réflexion auctoriale, subjective et donnée comme instantanée. Cette digression se caractérise par un décrochage énonciatif qui est un motif d'hétérogénéité entre le cadre-mère et le fragment digressif. Le deuxième emploi des parenthèses est particulier, car la parenthèse ouvrante est suivie du terme «verjus» - thème extrait du cadre explicatif-descriptif - et de la béquille argumentative «nota». On peut comparer cette combinaison de la parenthèse ouvrante avec le thème «verjus» à l'emploi des topicalisateurs comme marqueurs de digression (Quant au verjus, notez que...).

Cet exemple nous permettra de passer à la dernière catégorie de marqueurs explicites signalant l'inscription du digressif dans l'explicatif-argumentatif.

18. Ce marquage sous forme de mise en italique est attesté dès la première édition de 1600 (Paris, J. Métayer, 1004 p.). 


\subsubsection{Les béquilles argumentatives}

Une particularité du langage didactique en moyen français est l'utilisation du latin dans le corps du texte. Il s'agit souvent de petits mots, sortes de béquilles de l'argumentation (item, nota), qui «peuvent ponctuer le texte didactique et nuancer les articulations logiques du développement, toujours énumératif et encyclopédique dans ces passages» (Ueltschi, 2005 : 396-397). Comme en témoigne l'exemple [11], nota annonce une précision en marge du sujet principal (une déviation de la procédure vers le produit). La béquille item, quant à elle, ne fait pas dévier l'axe logique donné; elle le prolonge en accumulant des fragments discursifs qui s'inscrivent dans le cadre-mère mais en le remplissant au maximum. Les exemples suivants illustrent ces fonctionnements discursifs différents :

[12] $\{25\}$ Minces en Karesme est le regaing du chou, et durent jusques en mars; et lors sont icelles minces en mars de plus fort saveur a mengier. Et pour ce les convient plus parboulir, et en icelluy temps l'en arrache les trouz de terre.

$\{26\}$ Nota que en juillet quant il pleut l'en doit planter des choux.

$\{27\}$ Nota que se fromis habondent en ung jardin et l'en gecte en leur repaire de la cyeure d'aiz de chesne, ilz mourront ou wideront a la premiere pluye qui cherra; car les cyeures retiennent la moicteur.

$\{28\}$ Avril [...].

(Le mesnagier de Paris, II, ii, «Le jardinage»)

'\{25\} On appelle minces de carême la seconde pousse du chou. Elles durent jusqu'en mars; les choux de remontée de mars sont de saveur plus forte, aussi les fait-on bouillir plus longtemps; c'est à ce moment-là qu'on arrache les racines des choux restés en terre. \{26\} Nota que c'est en juillet quand il pleut qu'on doit planter les choux. \{27\} Nota que si les fourmis prolifèrent dans un jardin, il faut verser dans la fourmilière de la sciure de bois de chêne: elles mourront ou partiront dès la première pluie car la sciure retient l'humidité. \{28\} Avril [...].'

[13] \{947\} Le .3. signe, qui est veu plus certainement que le .2., est que le pacient doit tenir bien fort un fil ciré entre ses dens, et puis traire aucun ses ongles bien fort environ cel fil, et sachiés que le navré ou cran ne puet souffrir tel traiement sus le fil. \{948\} (Item autre signe: a ce prenés feives frasées, si les cuisiés bien en eaue, aussi com pour estre mengies, et reez la teste moult bien, si metés cest emplastre sus toute ceste partie de la teste, ainsi qu'ele soit bien couverte; si le lessiés ainsi estre jusques a l'endemain, et puis l'ostés soutilment o grant diligence; et ou vous verres que il ara entremesleure en l'emplastre et n'est mie si onnié la com allieurs, sachiés que le test. est la brisiés.)

\{949\} (Item autant fait v[i] erge chire emplastree et mise sus le chief, et endroit la briseure est la cire entresmellee et vergelee.)

(H. de Mondeville, La cbirurgie de maître Henri de Mondeville [début du XIV siècle], fragments 947-949, «.3. Signes de la froisseure du cran sans plaie de char de chief par dehors») 
' $\{947\}$ Le troisième signe qui est plus facilement identifiable que le second, se manifeste quand le patient doit tenir bien fort un fil ciré entre ses dents et ensuite tirer sur ce fil en le tenant entre ses ongles. Sachez que le patient blessé au crâne ne peut pas supporter une telle action. \{948\} (Item autre signe: prenez des fèves lissées, faites les bien cuire dans l'eau, comme si c'était pour un plat, rasez bien la tête et mettez cet emplâtre sur toute cette partie de la tête [...]. Laissez-le agir ainsi jusqu'au lendemain, et après vous l'enlevez avec adresse et application; et là où vous constaterez des infiltrations dans l'emplâtre et là où ce dernier n'est pas aussi régulier comme ailleurs, sachez que c'est bien là où le crâne est fracturé.) \{949\} (Item: le même effet est obtenu de la cire mise en emplâtre sur la tête, et à l'endroit de la fracture la cire est entremêlée et rayée.)'

(notre trad.)

Le nota de l'exemple [12] introduit des digressions car les précisions apportées s'éloignent du développement principal (le jardinage selon le calendrier de l'année). Le paragraphe $\{26\}$ interrompt la liste des travaux à réaliser au mois de mars. Le fragment $\{27\}$ introduit une rupture dans le traitement discursif du thème des choux et semble extrait de la chronologie mise en place. Il s'agit de deux déviations (digressions thématiques) produites par des glissements à partir d'un élément lexical figurant dans le fragment précédent ( $\{25\}$ arracher $>\{26\}$ planter; $\{26\}$ quant il pleut $>\{27\}$ a la premiere pluye). La relation de congruence entre les faits dénotés reste donc possible.

Quant à l'emploi de la béquille argumentative item, l'extrait de La cbirurgie de Henri de Mondeville ${ }^{19}$ s'inscrit dans cette logique de l'accumulation qui caractérise le marqueur en question. Après avoir indiqué les trois tests principaux permettant de diagnostiquer une fracture du crâne, Mondeville ajoute deux autres tests (signes) qui s'inscrivent dans l'axe logique donné. Contrairement à l'exemple [12], les parenthèses ne délimitent pas une digression mais signalent le statut secondaire des signes évoqués dans les fragments $\{948\}$ et $\{949\}$.

\section{Conclusion}

Qu'elle relève du docere ou du movere, la digression est une amplification qui consiste à inclure dans les textes des zones d'hétérogénéité. L'inscription de ces zones dans une séquence fonctionnant comme cadre-mère relève d'une stratégie destinée à emporter la conviction. Même si la mise en place de cette stratégie va de pair avec l'interruption du discours-cadre, le passage digressif conserve toujours des liens avec ce dernier. C'est à travers ces liens que se manifeste la cohérence de l'ensemble formé par le cadre et le détour. La matérialisation explicite de ces liens participe aux faits de stratification du discours. En effet, l'ordre rigoureux observé au niveau de la

19. Le traité, écrit entre 1306 et 1320 par Henri de Mondeville, fut traduit en 1314, peut-être par un jeune écolier normand qui commençait ses études de médecine à Paris. 
mise en place de la trame thématique et de la macrostructure du discours explicatif (dénombrement des propositions, paragraphes, chapitres) attribue à chaque séquence sa place dans la logique de l'ensemble tout en permettant à cette dernière de s'interrompre en vue de l'intercalation d'un développement accessoire. Les textes à dominante explicative/argumentative accueillent ces zones d'hétérogénéité qui se désolidarisent du discours-cadre afin d'y introduire des anecdotes, des réflexions ou considérations qui paraissent hors du sujet principal, mais qui vont pourtant au but essentiel.

Comme nous l'avons montré, l'usage de la digression permet paradoxalement de renforcer la cohésion de l'ensemble, qu'elle soit d'ordre intellectuel, moral ou historique. Le système de marqueurs à l'aide duquel la stratégie de digression se manifeste dans les textes permet de ponctuer le discours, de signaler le début et/ou la fin d'un enseignement, d'un divertissement, d'une réflexion. La digression relève toujours d'une stratégie discursive. C'est un acte conscient et dirigé, quel que soit le marqueur explicite ou l'embrayeur logique implicite convoqué pour l'introduire. Les détours provoqués peuvent être de nature thématique (par amplification d'un commentaire ponctuel) ou argumentative (par une rupture logique). Ils créent des espaces discursifs au sein desquels l'expression de la subjectivité va de pair avec le plaisir de la parole. Il s'agit donc d'une véritable mise en scène d'une frontière textuelle.

\section{Références bibliographiques}

ADAM, J.-M. 1992. Les textes, types et prototypes: récit, description, argumentation, explication et dialogue. Paris: Nathan.

Adam, J.-M. 2002. Cohérence. In P. Charaudeau et D. Maingueneau (éd.), Dictionnaire d'analyse $d u$ discours. Paris: Seuil: 99-100.

Adam, J.-M. et Heidmann, U. 2004. Des genres à la généricité. L’exemple des contes (Perrault et les Grimm). Langages 38 (153) : 62-72.

Beaugrande, R.-A. De 1979. Text and Sentence in Discourse Planning. In J. S. Petöfi (éd.), Text vs Sentence. Basic Questions of Text Linguistics. Hambourg: Buske : 467-494.

Bellert, I. 1970. On a Condition of the Coherence of Texts. Semiotica 2 (4): 335-363.

Brémond, C., Le Goff, J. et Schmitt, J.-C. 1982. L'exemplum. Turnhout: Brepols.

Charaudeau, P. et Maingueneau, D. (éd.) 2002. Dictionnaire d'analyse du discours. Paris : Seuil.

Charolles, M. 1978. Introduction aux problèmes de la cohérence des textes. Langue française $38: 7-41$.

Charolles, M. 1988. Les études sur la cohérence, la cohésion et la connexité textuelles depuis la fin des années 1960. Modèles linguistiques 10 (2): 45-66.

Charolles, M. 1995. Cohésion, cohérence et pertinence du discours. Travaux de linguistique 29: 125-151.

Chklovski, V. 1973. Sur la théorie de la prose. Lausanne: L’Âge d'Homme.

Coltier, D. 1986. Approches du texte explicatif. Pratiques: linguistique, littérature, didactique $51: 3-22$. 
Combettes, B. 2012a. Linguistique textuelle et diachronie. In F. Neveu, V. Muni Toke, P. Blumenthal, T. Klingler, P. Ligas, S. Prévost et S. Teston-Bonnard (éd.), SHS Web of Conferences. Actes du $3^{e}$ congrès mondial de Linguistique française - CMLF 2012 (Lyon, 4-7 juillet 2012). Les Ulis: EDP Sciences. Vol. 1: 3-10. En ligne à l'adresse suivante: https://www.shs-conferences.org/articles/shsconf/pdf/2012/01/shsconf_ cmlf12_000344.pdf.

Combettes, B. 2012b. Émergence et linguistique du texte. L'information grammaticale $134: 23-29$.

Combettes, B. et Tomassone, R. 1988. Le texte informatif: aspects linguistiques. Bruxelles: De Boeck-Wesmael.

Connochie-Bourgne, C. (éd.) 2005. La digression dans la littérature et l'art du Moyen Âge. Aix-en-Provence: Publications de l'université de Provence.

Connochie-Bourgne, C. 2006. Mise en récit et discours scientifique: les encyclopédies du $\mathrm{XIII}^{\mathrm{e}}$ siècle en langue vulgaire. In C. ThOMAsset (éd.), L'écriture du texte scientifique au Moyen Âge. Paris: Presses de l'université Paris-Sorbonne: 117-131.

Ducrot, O. et Schaeffer, J.-M. 1995. Nouveau dictionnaire encyclopédique des sciences du langage. Paris: Seuil.

Dupriez, B. 1984. Gradus. Les procédés littéraires. Paris: Union générale d'édition.

Gardes-Tamine, J. 2002. La rhétorique. Paris: A. Colin.

GrIzE, J.-B. 1981. Logique naturelle et explication: introduction. Revue européenne des sciences sociales 19 (56) : 7-14.

Halliday, M. A. K. et Hasan, R. 1976. Cohesion in English. Londres - New York: Longman.

JuILlARD, M. 2005. Avatars de la cohésion dans les corpus. Ordre des mots et coordonnants : variations génériques. In A. JAUBERT (éd.), Cohésion et cohérence. Études de linguistique textuelle. Lyon: ENS Éditions: 175-194.

Magri-Mourgues, V. 2005. Hétérogénéité, cohésion et cohérence: le statut de l'anecdote digressive dans un récit de voyage. In A. JAUBERT (éd.), Cohésion et cohérence. Études de linguistique textuelle. Lyon: ENS Éditions: 157-173.

Milhe Poutingon, G. 2012. Poétique du digressif: la digression dans la littérature de la Renaissance. Paris: Classiques Garnier.

Morier, H. 1981. Dictionnaire de poétique et de rbétorique. Paris: PUF.

Perelman, C. et Olbrechts-Tyteca, L. 1976. Traité de l'argumentation: la nouvelle rhétorique. Bruxelles: Éditions de l'Université de Bruxelles [1 ${ }^{\text {re }}$ éd. 1958: Paris: PUF].

SABRY, R. 1992. Stratégies discursives: digression, transition, suspens. Paris: Éditions de l'EHESS.

Silvi, C. 2003. Science médiévale et vérité: étude linguistique de l'expression du vrai dans le discours scientifique en langue vulgaire. Paris: $\mathrm{H}$. Champion.

Slakta, D. 1975. L'ordre du texte. Études de linguistique appliquée 19: 30-42.

Ueltschi, K. 2005. La digression dans l'économie du discours didactique vernaculaire du Moyen Âge français: manifestations et enjeux dans Le mesnagier de Paris. In C. Connochie-Bourgne (éd.), La digression dans la littérature et l'art du Moyen Âge. Aix-en-Provence: Publications de l'université de Provence: 391-407. 


\section{Textes cités et base textuelle interrogée}

ATILF (laboratoire Analyse et traitement informatique de la langue française) 2015. Dictionnaire du moyen français - DMF 2015. ATILF - CNRS - Université de Lorraine. En ligne à l'adresse suivante: http://www.atilf.fr/dmf.

Le mesnagier de Paris. G. E. Brereton et J. M. Ferrier (éd.), K. Ueltschi-Courchinoux (trad.) 1994. Paris: Librairie générale française [éd. bilingue: ancien français/ français moderne].

Mondeville, H. De, La chirurgie de maître Henri de Mondeville. A. Bos (éd.) 1897-1898. Paris: F. Didot.

Paré, A., Animaux, monstres et prodiges. C. GréGory (éd.) 1954. Paris: Club français du livre. Placides et Timéo ou li secrés as philosophes. C. Thomasset (éd.) 1980. Genève - Paris: Droz - H. Champion.

SERRES, O. DE 1600. Le théâtre d'agriculture et mesnage des champs. Paris: J. Métayer. 